ARTIGO ORIGINAL

ORIGINAL ARTICLE

\section{A capacidade de leitos hospitalares no Brasil, as internações no SUS, a migração demográfica e os custos dos procedimentos}

\author{
Hospitals beds in Brazil, SUS inpatients, demographic \\ migration and procedures costs
}

Beny José Finkelstein', Laerte Honorato Borges Junior

DOI: 10.21115/JBES.v12.n3.p273-80

\section{Palavras-chave}

SUS, leitos hospitalares, dados de vida real, gestão hospitalar, saúde pública, economia hospitalar

\section{Keywords}

SUS, hospital beds, real world data, real world evidences, hospital management, public health, hospital economy

\section{RESUMO}

Objetivo: Avaliar, por meio de dados de vida real, a capacidade dos leitos hospitalares brasileiros versus as recomendações da Organização Mundial de Saúde (OMS), como esses leitos estão sendo utilizados, de onde vêm os pacientes, quanto isso custa, as diferentes patologias que têm chegado aos hospitais e o percentual de tempo de internação em leitos de cuidado intensivo. Métodos: Estudo retrospectivo histórico realizado entre julho de 2018 e junho de 2019 a partir de dados obtidos do Datasus e subsequentemente processados em MySQL para diferentes perspectivas. Todos os índices foram construídos por meio de quintis de dias de internação e posteriormente subdivididos em análises mais específicas, confrontadas com literatura específica do assunto e diferentes diretrizes internacionais. Resultados: O Sistema Único de Saúde (SUS) teve um total de 9.275.680 pacientes únicos internados durante o período de análise, tendo um custo total de R\$ 183 bilhões, totalizando 63.817.613 de diárias hospitalares com uma média de 6,3 dias de internação, $R \$ 1.972,73$ de custo médio por internação e $\mathrm{R} \$ 286,73$ de custo médio diário. Conclusão: O SUS e toda a sua estrutura em perspectiva histórica são bem recentes. Cabe ainda aprimorar os processos de atenção de rede primária (portas de entrada), bem como desenvolver e disseminar os processos de desospitalização (portas de saída/reintrodução para a atenção primária).
Recebido em: 03/03/2020. Aprovado para publicação em: 27/11/2020.

Afiliação dos autores: Sem filiação a instituições de ensino ou instituições privadas para a execução deste trabalho. Nome da instituição onde o trabalho foi executado: $O$ trabalho foi executado em São Paulo e Uberlândia.

Informações sobre auxílios recebidos sob a forma de financiamento: Não houve financiamento para este trabalho. Conflito de interesses: Ambos os autores declaram trabalhar para a empresa ResMed LATAM.

Autor correspondente: Beny Finkelstein. Rua Canário, 988, 62. E-mail: benyfink@gmail.com / Laerte Honorato. Rua Antônio Francisco Rosa, 231. E-mail: laerteh@yahoo.com.br 


\section{Introdução}

A Política Nacional de Atenção Hospitalar (PNHOSP) no âmbito do Sistema Único de Saúde (SUS) foi publicada em dezembro de 2013 e estabelece as diretrizes para a organização do componente hospitalar na Rede de Atenção à Saúde (RAS). Essas diretrizes visam à garantia de universalidade de acesso, equidade e integralidade na atenção hospitalar, regionalização da atenção hospitalar, continuidade do cuidado por meio da articulação do hospital com os demais pontos de atenção da RAS, modelo de atenção centrado no cuidado ao usuário, de forma multiprofissional e interdisciplinar, acesso regulado, atenção humanizada, gestão de tecnologia em saúde, garantia da qualidade da atenção hospitalar e segurança do paciente, garantia da efetividade dos serviços, com racionalização da utilização dos recursos, financiamento tripartite, garantia da atenção à saúde indígena, transparência e eficiência na aplicação de recursos, participação do controle social e monitoramento e avaliação (Brasil, 2013).

Mesmo a PNHOSP estando em vigor desde 2013, grande parte dos problemas estruturais e históricos da rede hospitalar não foram resolvidos. Apesar de várias mudanças terem acontecido, continua a dificuldade de lidar com o aumento da demanda assistencial, a quantidade de leitos disponíveis, o permanente subfinanciamento do SUS e vários outros desafios (Mendes, 2001).

Nesse sentido, é importante analisar as políticas públicas e processos de gestão que interferem no acesso e na disponibilidade de leitos hospitalares, os custos operacionais para mantê-los ativos e o perfil epidemiológico das internações atuais, e espera-se que essas análises possam contribuir com evidências científicas aplicáveis. Estudos avaliativos de políticas públicas, quando direcionados à gestão, podem contribuir para meIhorar a efetividade das estratégias e ações planejadas, assim como subsidiar a tomada de decisões dos gestores (Teixeira, 2010; Tanaka \& Ribeiro, 2017). Assim, faz sentido avaliar a capacidade instalada de internações no sistema público de saúde e analisar como estão sendo utilizadas pelos usuários considerando o tempo médio de permanência em enfermarias e unidades de terapia intensiva (UTI), a procedência dos pacientes por porte de municípios, as principais causas de doenças que estão gerando as internações e o custo total para o SUS.

\section{Métodos}

Mediante a extração de bases do Datasus (CNES, SIH e SIGTAP), foram realizadas análises dos bancos de dados retrospectivos, identificando pacientes únicos por meio de uma codificação determinada e acompanhando seu itinerário durante 12 meses a partir de uma internação inicial. Essas análises foram a soma dos dias de internação de cada um desses pacientes únicos determinados, por meio de estratificações geográficas, de faixas de diárias de internação e patologia, sejam absolutas, percentuais ou percentis. Essas análises buscaram o menor conjunto (chave de paciente único) para identificar o local de residência do paciente e as diferentes patologias que o acometeram durante o período de julho de 2018 a junho de 2019 (com consulta realizada nos bancos de dados em agosto de 2019), e foram processadas utilizando o software TabWin e MySQL mediante a estruturação de arquitetura de dados respectiva.

Os dados extraídos foram organizados em diferentes tabelas analíticas; sendo a primeira a de faixa de diárias de internação, quantidade de pacientes únicos e seus custos associados; a segunda a de porcentagem de utilização de serviço de terapia intensiva para os grupos das maiores faixas de diárias de internação; a terceira com a mesma informação anterior com a identificação da unidade federativa onde foi realizada a internação; a quarta com os principais motivos para internação prolongada agrupados por classificação de CID; a quinta com o detalhamento do grupo de CID e priorização dessas causas de internação.

Foi necessário também realizar a estratificação desses números e avaliar a possibilidade de otimização do complexo hospitalar brasileiro. Portanto, foram realizadas separadamente em quintis acumulados de dias de internação em diferentes grupos, sendo esses de 0 a 6 dias, de 7 a 16 dias, de 17 a 31 dias, de 32 a 60 dias e mais de 61 dias, observando-se o somatório total dos dias internados. Por esse motivo, os grupos tiveram dias similares de internação, com menor quantidade de pacientes. Todos esses grupos com pacientes que tiveram internações entre 0 e 6 dias têm custos e médias de permanência diferentes daqueles grupos que permaneceram mais de 61 dias internados.

Todos os grupos foram estratificados em três movimentações geográficas, sendo elas: 01 - Mesmo município, que significa que o paciente reside no mesmo município onde foi atendido; 02 - Maior que 150 mil, significando que o paciente não reside no mesmo município em que foi atendido, porém o município em que ele reside tem mais de 150 mil habitantes; 03 - Menor que 150 mil, que significa que o paciente não reside no mesmo município no qual foi atendido e o seu município possui menos de 150 mil habitantes.

\section{Resultados}

Segundo dados do próprio sistema de saúde, no Brasil existem cerca de 493 mil leitos de diferentes modalidades disponíveis para atendimento da população brasileira (CNES, 2019), gerando uma relação de 2,3 leitos/mil habitantes, porém o valor preconizado pela Organização Mundial de Saúde (OMS) é de 3 leitos/mil habitantes. Na Tabela 1, estratifica-se o número de leitos total, públicos e privados, a relação do número para cada mil habitantes por região de saúde e o número de leitos necessários para chegar à relação preconizada pela OMS. Destacam-se a necessidade da obtenção de leitos no setor público e a sobra dos leitos privados, ajustados para a população que acessa cada um desses serviços. 
Observando os dados do sistema de atenção hospitalar tabela, foi levantado e analisado o perfil das internações no acumulado de 12 meses (julho 2018 a junho 2019). Nesse período, foram internados 9,3 (SIH, 2019) milhões de usuários do SUS, e eles tiveram um custo de 183 bilhões (SIH, 2019) para os cofres públicos da União, com base na tabela de repasses do Sigtap. A média foi de 6,9 dias por internação, com um custo médio aproximado de R\$ 1.972,73 (SIH, 2019).

Na Tabela 2, foi estratificado o número total de pacientes internados pelos dias de internação, com seu total de dias, média de dias internado, valor médio por internação e origem do paciente por porte populacional do município.

Tabela 1. Número total de leitos disponíveis no sistema de saúde público e privado com a relação do número de leitos para cada mil habitantes e do número de leitos necessários para atingir o preconizado pela Organização Mundial de Saúde

\begin{tabular}{|c|c|c|c|c|c|c|c|c|c|c|c|c|}
\hline \multirow[b]{2}{*}{ Região Brasil } & \multicolumn{4}{|c|}{ População Total } & \multicolumn{4}{|c|}{ SUS DEPENDENTE } & \multicolumn{4}{|c|}{ SISTEMA SUPLEMENTAR } \\
\hline & $\begin{array}{l}\text { LEITOS } \\
\text { TOTAL }\end{array}$ & $\begin{array}{c}\text { População } \\
2020\end{array}$ & $\begin{array}{c}\text { Leitos } \\
\text { Total } \\
\text { mil } \\
\text { hab }\end{array}$ & $\begin{array}{c}\text { Dif. Leitos } \\
\text { objetivo }\end{array}$ & LEITOS & $\begin{array}{c}\text { POP. } \\
\text { SUS DEP. }\end{array}$ & $\begin{array}{l}\text { Leitos } \\
\text { SUS } \\
\text { mil } \\
\text { hab }\end{array}$ & $\begin{array}{c}\text { Dif. } \\
\text { Leitos } \\
\text { objetivo }\end{array}$ & $\begin{array}{c}\text { LEITOS } \\
\text { PRIVADO }\end{array}$ & $\begin{array}{c}\text { POP. } \\
\text { PRIVADO }\end{array}$ & $\begin{array}{c}\text { Leitos } \\
\text { Priv } \\
\text { mil } \\
\text { hab }\end{array}$ & $\begin{array}{c}\text { Dif. Leitos } \\
\text { objetivo }\end{array}$ \\
\hline CENTRO-OESTE & 42.413 & 16.504 .303 & 2,57 & $(7.107)$ & 25.938 & 13.314 .466 & 1,9 & $(14.005)$ & 16.475 & 3.189 .837 & 5,2 & 6.905 \\
\hline NORDESTE & 125.769 & 57.374 .243 & 2,19 & $(46.731)$ & 99.029 & 50.782 .692 & 2,0 & $(53.319)$ & 26.740 & 6.591 .551 & 4,1 & 6.965 \\
\hline NORTE & 35.279 & 18.672 .591 & 1,89 & $(21.171)$ & 26.732 & 16.977.108 & 1,6 & (24.199) & 8.547 & 1.695 .483 & 5,0 & 3.461 \\
\hline SUDESTE & 208.197 & 89.012 .240 & 2,34 & $(59.803)$ & 120.952 & 60.353 .729 & 2,0 & $(60.109)$ & 87.245 & 28.658 .511 & 3,0 & 1.269 \\
\hline SUL & 81.352 & 30.192 .315 & 2,69 & $(10.648)$ & 56.002 & 23.319 .404 & 2,4 & $(13.956)$ & 25.350 & 6.872 .911 & 3,7 & 4.731 \\
\hline Total & 493.010 & 211.755 .692 & 2,3 & (145.460) & 328.653 & 164.747 .399 & 2,0 & (165.589) & 164.357 & 47.008 .293 & 3,5 & 23.332 \\
\hline
\end{tabular}

Fonte: CNES 201908 (Cadastro Nacional de Estabelecimentos de Saúde).

Tabela 2. Estratificação do número total de pacientes internados pelos dias de internação, com seu total de dias, média de dias internado, valor médio por internação e origem do paciente por porte populacional do município

\begin{tabular}{|c|c|c|c|c|c|c|c|}
\hline INTERNAÇÃO & $\begin{array}{l}\text { MUNICÍPIO DE } \\
\text { ORIGEM }\end{array}$ & $\begin{array}{l}\text { QDE DE } \\
\text { PACIENTES }\end{array}$ & $\frac{\text { VALOR TOTAL }}{\text { (em milhão) }}$ & $\begin{array}{l}\text { DIAS TOTAIS } \\
\text { INTERNAÇÃO }\end{array}$ & $\begin{array}{l}\text { MÉDIA DIAS } \\
\text { INTERNADO }\end{array}$ & $\begin{array}{l}\text { VALOR MÉDIO } \\
\text { INTERNAÇÃO }\end{array}$ & $\begin{array}{l}\text { MÉDIA CUSTO } \\
\text { DIÁRIO }\end{array}$ \\
\hline \multirow[t]{3}{*}{ 00-06 } & 01 - Mesmo Município & 3.155 .579 & $R \$ 2.362,12$ & 7.761 .913 & 2,5 & $R \$ 748,55$ & $R \$ 304,32$ \\
\hline & 02 - Maior que $150 \mathrm{mil}$ & 1.328 .903 & $R \$ 1.283,89$ & 3.381 .449 & 2,5 & $R \$ 966,13$ & $R \$ 379,69$ \\
\hline & 03 - Menor que $150 \mathrm{mil}$ & 2.561 .999 & $R \$ 2.477,94$ & 6.335 .959 & 2,5 & $R \$ 967,19$ & $\mathrm{R} \$ 391,09$ \\
\hline 00-06 Total & & 7.046 .481 & $R \$ 6.123,95$ & 17.479 .321 & 2,5 & $R \$ 869,08$ & $R \$ 350,35$ \\
\hline \multirow[t]{3}{*}{ 07-16 } & 01 - Mesmo Município & 582.012 & $R \$ 1.226,56$ & 5.823 .217 & 10 & $R \$ 2.107,45$ & $R \$ 210,63$ \\
\hline & 02 - Maior que $150 \mathrm{mil}$ & 338.224 & $R \$ 959,52$ & 3.465 .620 & 10,2 & $R \$ 2.836,93$ & $R \$ 276,87$ \\
\hline & 03 - Menor que $150 \mathrm{mil}$ & 499.785 & $\mathrm{R} \$ 1.551,71$ & 5.097 .193 & 10,2 & $R \$ 3.104,76$ & $R \$ 304,43$ \\
\hline 07-16 Total & & 1.420 .021 & $R \$ 3.737,80$ & 14.386 .030 & 10,1 & $R \$ 2.632,21$ & $\mathrm{R} \$ 259,82$ \\
\hline \multirow[t]{3}{*}{$17-31$} & 01 - Mesmo Município & 180.430 & $R \$ 935,69$ & 3.989 .014 & 22,1 & $R \$ 5.185,89$ & $R \$ 234,57$ \\
\hline & 02 - Maior que $150 \mathrm{mil}$ & 128.301 & $R \$ 821,72$ & 2.903 .826 & 22,6 & $R \$ 6.404,62$ & $R \$ 282,98$ \\
\hline & 03 - Menor que 150 mil & 175.434 & $\mathrm{R} \$ 1.245,47$ & 4.015 .307 & 22,9 & $R \$ 7.099,34$ & $R \$ 310,18$ \\
\hline 17-31 Total & & 484.165 & $R \$ 3.002,87$ & 10.908 .147 & 22,5 & $R \$ 6.202,17$ & $R \$ 275,29$ \\
\hline \multirow[t]{3}{*}{$32-60$} & 01 - Mesmo Município & 79.533 & $\mathrm{R} \$ 851,50$ & 3.272 .444 & 41,1 & $R \$ 10.706,20$ & $R \$ 260,20$ \\
\hline & 02 - Maior que $150 \mathrm{mil}$ & 63.331 & $\mathrm{R} \$ 790,97$ & 2.687 .966 & 42,4 & $R \$ 12.489,49$ & $R \$ 294,26$ \\
\hline & 03 - Menor que 150 mil & 83.061 & $R \$ 1.127,95$ & 3.562 .117 & 42,9 & $\mathrm{R} \$ 13.579,79$ & $\mathrm{R} \$ 316,65$ \\
\hline 32-60 Total & & 225.925 & $R \$ 2.770,42$ & 9.522 .527 & 42,1 & $R \$ 12.262,56$ & $R \$ 290,93$ \\
\hline \multirow[t]{3}{*}{$61+$} & 01 - Mesmo Município & 32.699 & $\mathrm{R} \$ 786,47$ & 3.740 .845 & 114,4 & $\mathrm{R} \$ 24.051,86$ & $R \$ 210,24$ \\
\hline & 02 - Maior que $150 \mathrm{mil}$ & 30.221 & $\mathrm{R} \$ 852,11$ & 3.656 .881 & 121 & $R \$ 28.195,87$ & $R \$ 233,01$ \\
\hline & 03 - Menor que $150 \mathrm{mil}$ & 36.168 & $R \$ 1.024,80$ & 4.123 .862 & 114 & $\mathrm{R} \$ 28.334,52$ & $\mathrm{R} \$ 248,51$ \\
\hline $61+$ Total & & 99.088 & $R \$ 2.663,38$ & 11.521 .588 & 116,3 & $R \$ 26.878,96$ & $\mathrm{R} \$ 231,16$ \\
\hline BRASIL TOTAL & & 9.275 .680 & $\mathrm{R} \$ 18.298,43$ & 63.817 .613 & 6,9 & $\mathrm{R} \$ 1.972,73$ & $\mathrm{R} \$ 286,73$ \\
\hline
\end{tabular}

Fonte: Datasus - SIH RD (julho 2018 a junho 2019). 
No grupo que permaneceu internado entre 0 e 6 dias, os pacientes tiveram um total de 7 milhões de pacientes únicos, permaneceram na média 2,5 dias internados, tiveram um custo médio de $\mathrm{R} \$ 869,1$ por internação e um custo médio por diária de $\mathrm{R} \$ 350,35$; enquanto o grupo que permaneceu 61 dias ou mais teve um total de 99.068 pacientes, um tempo médio de permanência de 116,3 dias (30\% de um ano), um valor médio de $\mathrm{R} \$ 26.878,96$ por internação e um custo médio por diária de $\mathrm{R} \$ 286,73$.

Nota-se que nesses extratos os pacientes que precisaram se deslocar entre seu município de residência e o município de atendimento têm um custo médio maior de R\$ 4.200 por internação ( $R \$ 24.051$ versus 28.334.52), não havendo diferenças a nível Brasil entre diferença de municípios maiores ou menores de 150 mil habitantes.

Na Figura 1 constam os grupos com mais tempo de internação (entre 32 e 60 dias e 61 dias ou mais) e a porcentagem de dias em UTI ou unidade de cuidados intermediários (UCI) com outros leitos.

Nota-se que a composição em nível de Brasil (média de todos os 325.023 pacientes internados) é de 15,3\% dias em UTI ou UCl, havendo uma diferença entre os pacientes com internações entre 32 e 60 e 61 dias ou mais, com 17\% e 14\%, respectivamente.

Na Figura 2, constam a estratificação dos grupos com internações mais longas (entre 32 e 60 dias e 61 dias ou mais) e a porcentagem de dias em UTI ou UCI com outros leitos nos diferentes estados brasileiros.

Essa diferença também pode ser encontrada em cada um dos estados da federação. Para o grupo de pacientes que permaneceram 32 a 60 dias internados, os estados do Ceará, Mato Grosso, Pará, Paraíba e Mato Grosso do Sul são aqueles que tiveram mais de $20 \%$ do tempo de internação total em UTI e UCl, totalizando uma média de 12 dias de UTI. Estados como Tocantins, Maranhão, Roraima e Rio Grande do Norte tiveram uma média de internação entre 10\% e 13\%, sendo respectivos a seis dias.

Já quando observado o grupo de pacientes que ficou internado 61 dias ou mais (média de 116,3 - Tabela 1), nota-se que os estados da Paraíba, Pará, Sergipe, Rio Grande do Sul e São Paulo são aqueles que mantêm uma média maior de $17 \%$. Destaca-se o estado da Paraíba, onde os pacientes permanecem $26 \%$ do tempo de internação em leitos de alta complexidade, um total de aproximadamente 29 dias contínuos.

Outro ponto fundamental para análise das internações são os motivos pelos quais os pacientes foram levados ao centro hospitalar. Para isso, foram trazidos os principais motivos da primeira internação dos pacientes, acumulados em 12 meses e também em nível de Brasil. A Tabela 3 traz essa análise.

Os principais motivos de internação para mais de 32 dias (e 61 dias ou mais), segundo o capítulo CID-10, são transtornos mentais e comportamentais, doenças do aparelho circulatório, algumas doenças infecciosas e parasitárias e, por fim doenças, do aparelho respiratório; estas representam 51\% de todas as internações acima de 32 dias. A lista dos 10 principais motivos representa quase $90 \%$ de todas as internações do Brasil, tendo uma média de 16\% desse período em leitos de UTI/UCl. O grupo de pacientes que ficou internado por doenças do aparelho respiratório permaneceu uma média de 25\% do período internado em leitos de UTI/UCl.

Ainda, buscou-se detalhar entre os capítulos, em nível do grupo, ou o principal motivo de diagnóstico, a fim de detaIhar a informação. Na Tabela 4, foram excluídos das análises os pacientes que são dos grupos CID-10 (F) - Motivos psiquiátricos, pois, além de utilizarem leitos diferentes, eles não permanecem na UTI durante nenhum período, como visto acima.

A lista desses 12 principais motivos de internação com utilização de UTI e UCI representa, para o número total de pacientes, um total de $27 \%$ e $24 \%$ para os grupos de 32 a 60 e 61 dias ou mais, respectivamente; já para a quantidade de diárias totais, representa um total de $27 \%$ e $19 \%$, para os grupos de 32 a 60 e 61 dias ou mais de internação, respectivamente; ainda assim estas poderiam ser agrupadas nos seus capítulos, tendo como os principais motivos de internação.

Enquanto o primeiro motivo de internação para 32 a 60 dias é de $J 18$ Pneumonia para Microog NE, este é o quarto motivo no grupo que permaneceu 61 dias ou mais internado.

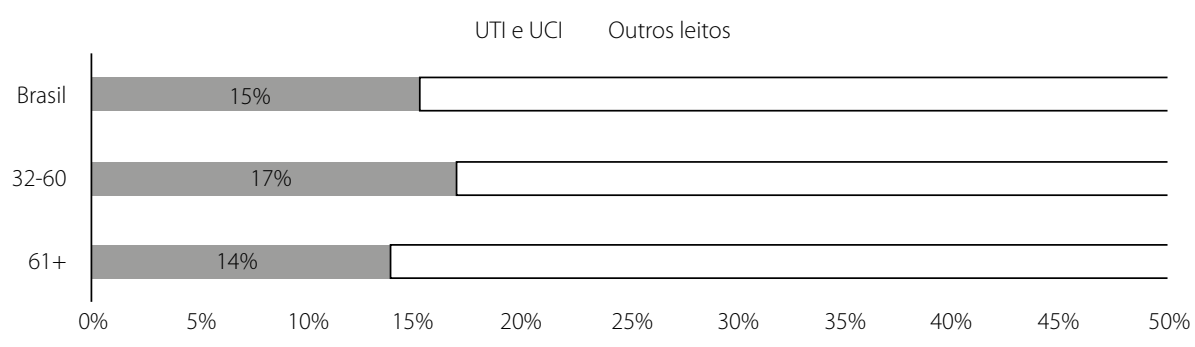

Fonte: Datasus - SIH RD (julho 2018 a junho 2019).

Figura 1. Porcentagem de internação em leitos de UTI e UCl, comparados com outros leitos, no grupo de pacientes internados de 32 a 60 dias e os de mais de 61 dias 
O grupo com 32 a 60 dias de internação teve 8.403 pacientes e ficou em leitos de UTI/UCI durante 18\% do período, ou seja, uma média de oito dias, enquanto o grupo que ficou internado 61 dias ou mais foi de 2.542 pacientes e ficou internado em leitos de UTI/UCI durante $26 \%$ do período, uma média de 30 dias.
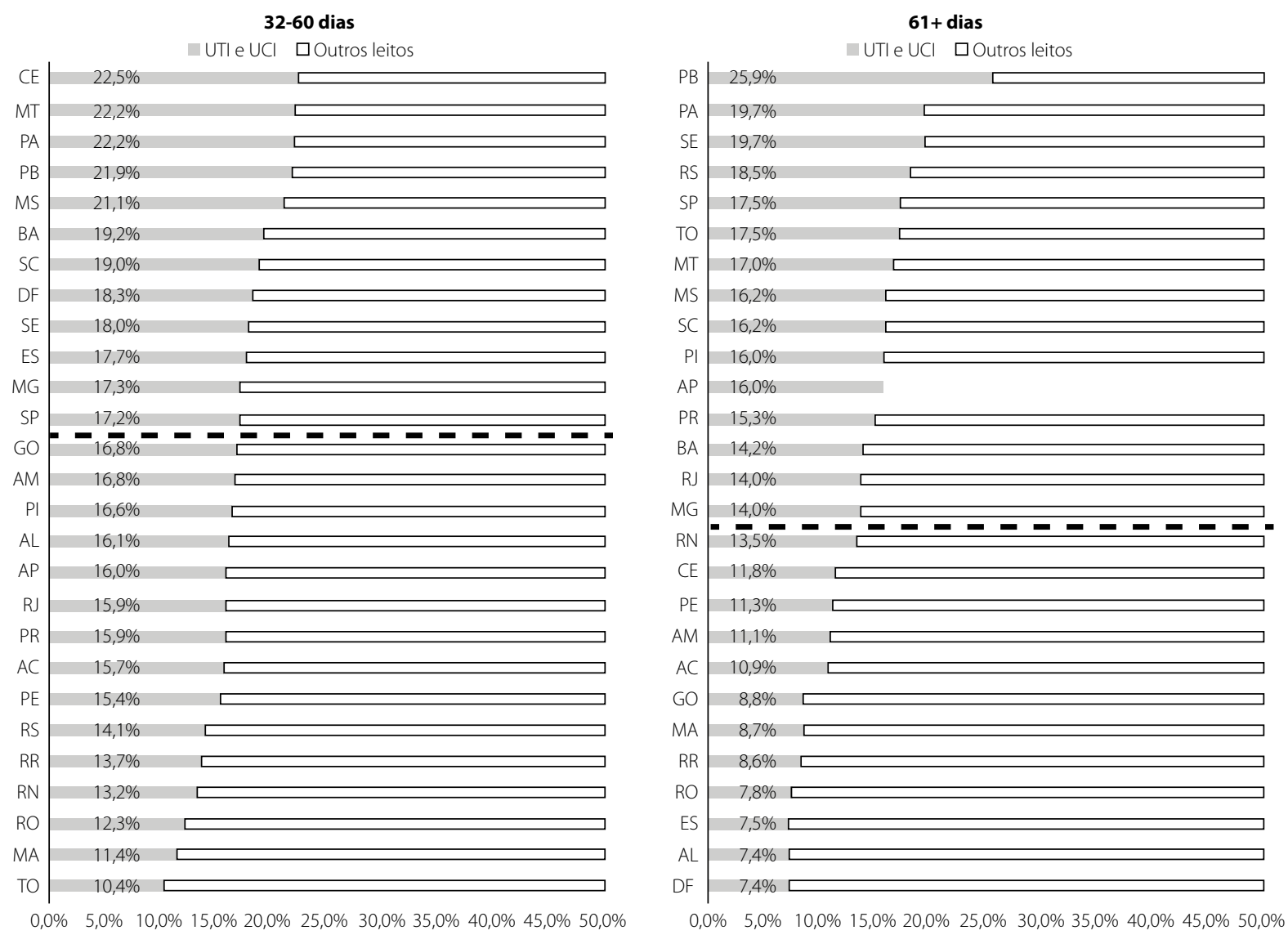

Fonte: Datasus - SIH RD (julho 2018 a junho 2019).

Figura 2. Porcentagem de internação em leitos de UTI e UCl, comparados com outros leitos, no grupo de pacientes internados de 32 a 60 dias e os de mais de 61 dias nos diferentes. estados brasileiros

Tabela 3. Classifica os principais motivos de internação e o local entre UTI/UCl e outro local

\begin{tabular}{|c|c|c|c|c|}
\hline RANK & Capítulo CID-10 & Diárias & Representatividade & INTERNAÇÃO UTI/UCI \\
\hline 1 & Transtornos mentais e comportamentais & 4.336 .302 & $21 \%$ & $0 \%$ \\
\hline 2 & Doenças do aparelho circulatório & 2.773 .861 & $13 \%$ & $17 \%$ \\
\hline 3 & Algumas doenças infecciosas e parasitárias & 1.798 .231 & $9 \%$ & $21 \%$ \\
\hline 4 & Doenças do aparelho respiratório & 1.725 .895 & $8 \%$ & $25 \%$ \\
\hline 5 & $\begin{array}{l}\text { Lesões, envenenamento e algumas outras consequências de causas } \\
\text { externas }\end{array}$ & 1.714 .473 & $8 \%$ & $14 \%$ \\
\hline 6 & Neoplasias (tumores) & 1.677 .158 & $8 \%$ & $9 \%$ \\
\hline 7 & Doenças do sistema nervoso & 1.583 .369 & $8 \%$ & $7 \%$ \\
\hline 8 & Algumas afecções originadas no período perinatal & 1.173 .732 & $6 \%$ & $72 \%$ \\
\hline 9 & Doenças do aparelho geniturinário & 1.009 .460 & $5 \%$ & $11 \%$ \\
\hline \multirow[t]{2}{*}{10} & Doenças do aparelho digestivo & 966.026 & $5 \%$ & $18 \%$ \\
\hline & Total & 18.758.507 & $89 \%$ & $16 \%$ \\
\hline
\end{tabular}

Fonte: Datasus - SIH RD (julho 2018 a junho 2019). 
Tabela 4. Classifica os principais motivos de internação com a exclusão dos motivos psiquiátricos, o rank de diária, tempo de permanência, número de diárias e porcentagem de internação em UTI/CTI

\begin{tabular}{|c|c|c|c|c|c|c|c|c|}
\hline \multirow{2}{*}{$\begin{array}{l}\text { CID-10 - PRIMEIRA INTERNAÇÃO } \\
\text { (Excluindo motivos psiquiátricos) }\end{array}$} & \multicolumn{2}{|c|}{$\begin{array}{l}\text { RANK DE } \\
\text { DIÁRIAS }\end{array}$} & \multicolumn{2}{|c|}{ \# Pacientes } & \multicolumn{2}{|c|}{ \# Diárias } & \multicolumn{2}{|c|}{$\begin{array}{c}\text { \% Internação } \\
\text { UTI/UCI }\end{array}$} \\
\hline & $32-60$ & $61+$ & $32-60$ & $61+$ & $32-60$ & $61+$ & $32-60$ & $61+$ \\
\hline J18 Pneumonia p/microorg NE & 1 & 4 & 8.403 & 2.542 & 348.026 & 236.620 & $18 \%$ & $26 \%$ \\
\hline I50 Insuf cardíaca & 2 & 5 & 7.571 & 2.500 & 317.210 & 221.835 & $18 \%$ & $20 \%$ \\
\hline A41 Outr septicemias & 3 & 1 & 6.793 & 2.969 & 287.191 & 285.268 & $35 \%$ & $36 \%$ \\
\hline P07 Transt rel gest curt dur peso baix nasc NCOP & 4 & 6 & 5.847 & 2.231 & 248.133 & 200.812 & $71 \%$ & $74 \%$ \\
\hline N18 Insuf renal crônica & 5 & 3 & 5.338 & 2.558 & 229.450 & 237.087 & $11 \%$ & $9 \%$ \\
\hline I64 Acid vasc cerebr NE como hemorrag isquêmico & 6 & 8 & 4.943 & 1.603 & 206.961 & 155.330 & $21 \%$ & $19 \%$ \\
\hline I21 Infarto agudo do miocárdio & 7 & 11 & 4.601 & 1.363 & 192.304 & 114.316 & $20 \%$ & $19 \%$ \\
\hline P22 Desconforto respirat do recém-nascido & 8 & 2 & 4.236 & 2.582 & 185.277 & 243.702 & $76 \%$ & $77 \%$ \\
\hline S72 Frat do fêmur & 9 & 12 & 4.065 & 1.111 & 168.029 & 97.344 & $9 \%$ & $10 \%$ \\
\hline J15 Pneumonia bacter NCOP & 10 & 10 & 3.817 & 1.185 & 158.760 & 117.584 & $19 \%$ & $24 \%$ \\
\hline A49 Infecc bacter de localiz NE & 11 & 9 & 3.618 & 1.488 & 152.950 & 146.760 & $17 \%$ & $23 \%$ \\
\hline J96 Insuf respirat NCOP & 12 & 7 & 2.141 & 1.295 & 91.256 & 160.633 & $40 \%$ & $42 \%$ \\
\hline TOTAL & & & 61.373 & 23.427 & 2.585 .547 & 2.217.291 & - & - \\
\hline Total por grupo analisado & & & 225.925 & 99.088 & 9.522 .527 & 11.521 .588 & - & - \\
\hline Representatividade total & & & $27 \%$ & $24 \%$ & $27 \%$ & $19 \%$ & - & - \\
\hline
\end{tabular}

Fonte: Datasus - SIH RD (julho 2018 a junho 2019).

\section{Discussão}

A média do Brasil de leitos para cada 1.000 habitantes é de 2,3. Considerando somente leitos do SUS, essa relação cai para 2 e no privado sobe para 3,5. Essa diferença reforça a necessidade de construção de aproximadamente 146 mil leitos para a população dependente do SUS nas diversas regiões do Brasil, enquanto na saúde suplementar sobram leitos, pois o preconizado pela OMS é de três leitos para cada 1.000 habitantes. Esse importante déficit no SUS tem sido um grande desafio para os gestores, pois os cofres públicos não conseguem acompanhar essa demanda de ampliação, bem como de manutenção e funcionamento (custeio), o que faz essa estratégia sair bem onerosa e ainda de médio e longo prazo, não solucionando o problema enfrentado (Mendes, 2001).

Um olhar panorâmico sobre a distribuição dos 493.010 leitos do conjunto dos hospitais brasileiros indica que $66,6 \%$ deles atendem exclusivamente o SUS, para 77,8\% da população brasileira. Os demais $22,2 \%$ da população que possui algum tipo de convênio de saúde tem disponíveis os 33,4\% do total de leitos disponíveis, que são os privados, além de toda a rede de hospitais públicos que têm acesso universal (Pereira Júnior, 2018). Esse desequilíbrio poderia ser minimizado caso houvesse uma maior contratualização de leitos privados para atendimento do SUS.

Uma das justificativas do Ministério da Saúde para esse cenário é que o país segue a tendência mundial de desospitalização, uma vez que, com os avanços tecnológicos, tratamentos que antes exigiam internação passaram a ser feitos no âmbito ambulatorial e domiciliar. Dados da Organização para a Cooperação e Desenvolvimento Econômico (OCDE) apontam que o próprio sistema inglês referência mundial de sistema público de saúde - reduziu em 30\% o número de leitos em 10 anos (Ramalho, 2018).

A desospitalização tem sido uma opção que tem se mostrado extremamente vantajosa no universo da saúde. No Brasil, esse conceito está se disseminando nos serviços de saúde por meio dos programas de "Serviço de Atenção Domiciliar" (SAD) no setor público de saúde, bem como por meio das chamadas home cares no setor privado (Silva et al., 2010). A principal vantagem é tratar dos pacientes num ambiente mais adequado à sua condição clínica, em um ambiente mais cômodo e evitando complicações e infecções hospitalares, além de ter menores custos operacionais (Silva et al., 2017). Ele surgiu para que os hospitais possam focar no atendimento dos casos agudos, cirúrgicos e de alta complexidade, com o objetivo de que os pacientes permaneçam internados no período em que eles necessitam de cuidado profissional e da tecnologia que o hospital oferece, associado às facilidades e recursos oferecidos pelas suas estruturas, além de melhor acesso e qualidade de cuidado com mais humanização da assistência. A partir do momento em que o paciente estabiliza sua condição clínica, a continuidade do cuidado é garantida e transferida para sua residência com 
o monitoramento de uma rede de apoio mais preparada e adequada para complementar o tratamento de pacientes em qualquer faixa etária, ou seja, adulto ou pediátrico e até mesmo aqueles com dependência de tecnologia como a ventilação mecânica (Carvalho et al., 2019).

A teoria dessa prática médica vem se consolidando e buscando uma redução do tempo de permanência em internações hospitalares. Recomenda-se que os pacientes voltem o mais rápido possível para o convívio familiar e social. O ambiente hospitalar passou a ser considerado potencialmente iatrogênico. O desenvolvimento científico e tecnológico permite a transferência de procedimentos para fora do hospital.

É seguindo essa nova tendência que desde 2011 o Ministério da Saúde criou o SAD no SUS com o nome Programa Melhor em Casa (Brasil, 2016). O SAD é um serviço complementar ou substitutivo ao atendimento ambulatorial ou à internação hospitalar; até o final de 2019 eram 1.118 equipes em funcionamento em 451 cidades e em 25 estados brasileiros e no final de dezembro de 2019 o Ministério da Saúde apostou mais uma vez na estratégia e habilitou novas 411 equipes, aumentando em 36\% a capacidade instalada para aumentar o número de leitos domiciliares.

Sobre o número e o custo das internações geradas no período analisado do estudo, foi possível identificar a diferença entre eles no grupo de 0 a 6 dias e no grupo acima de 30 dias. Para sanar essa discrepância nos valores de custeio e do tempo médio de permanência, são necessários medidas emergenciais de conscientização e melhores processos de trabalho integrado e intersetorial com todos os setores da RAS, para que se otimizem mais os recursos hospitalares, melhorando a regulação do acesso e das indicações de internação hospitalar e ampliando a porta de saída, para que, principalmente nos casos crônicos, seja feita a transição do cuidado para o domicílio e assistido por um outro ponto da rede, fazendo com que o ambiente hospitalar seja respectivos para o atendimento assistencial agudo e transitório, e não um lar para o paciente.

Muitas dificuldades encontradas nessa mudança de paradigma devem-se ao papel estratégico que os hospitais seguem ocupando na assistência à saúde. Mesmo com as transformações assistenciais e organizacionais ocorridas nos sistemas de saúde, com a ampliação da atenção primária, atenção especializada e oferta de serviços, a cultura de que os hospitais concentram melhores saberes e tecnologias faz com que os usuários e trabalhadores utilizem mais esse ponto da rede, mesmo sem critérios. Ainda é no hospital que a maioria das pessoas nascem, são cuidadas em situações mais graves e morrem (Braga Neto et al., 2012). O ambiente hospitalar é responsável pela maior parte dos gastos dos sistemas de saúde.

Sobre as análises do leito de UTI, pode ser observado que $85 \%$ são internações prolongadas; e esses leitos são os de maiores custos para o sistema. Muitas dessas internações hospitalares longas, principalmente as que ocorrem em UTI, são decorrentes de necessidades de tecnologias de alta complexidade, falta de cuidadores habilitados para garantir o cuidado após a alta hospitalar e vulnerabilidade e risco social aumentados, o que faz com que o usuário permaneça internado mesmo após a estabilização clínica, aumentando, assim, o tempo de permanência no hospital e reduzindo a disponibilidade de leitos (Panorama, 2015). Para muitos desses usuários, o hospital passa a ser o local de moradia, servindo como garantia de suporte ao cuidado, pois muitos serviços médicos não têm sequer conhecimento da existência do SAD nem de como podem oferecer esse tratamento em casa, com o suporte da RAS, gerando mais um importante problema no sistema público de saúde brasileiro (Oliveira Neto \& Dias, 2014). Essas hospitalizações prolongadas e as visitas frequentes aos serviços de pronto atendimento apresentam um alto custo para o sistema de saúde que pode ser evitado se elas forem substituídas por internações domiciliares, por meio dos SAD (Brasil, 2012).

Outro ponto a ser ressaltado nesta discussão é a utilização dos leitos domiciliares como complemento e/ou substituição aos leitos hospitalares dentro do SUS. Hoje, no programa Melhor em Casa do SAD, estão habilitadas 716 equipes para atendimento domiciliar, com uma média de 49 pacientes cada (Brasil, 2019), totalizando um montante de 35 mil leitos adicionais ao sistema de saúde, diminuindo a carência enfrentada atualmente. Portanto, à medida que as evidências científicas permitirem identificar e mapear problemas e oportunidades para uso e aplicação de soluções que investigam a efetividade e os custos nos leitos hospitalares, essas análises poderão auxiliar na seleção das intervenções mais resolutivas com menor custo e agregar elementos para alterações e aprimoramento das políticas de saúde, aumentando o acesso, a segurança e a qualidade dos serviços e do cuidado prestado. Com esse apoio científico mais consistente com uma vinculação mais próxima a atividades de interesse público, o setor de pesquisa se fortalece, obtendo mais reconhecimento, legitimidade e recursos, atraindo mais talentos e competência.

\section{Conclusão}

O SUS, tanto na perspectiva pública como na suplementar privada, está em constante alteração e amadurecimento. O SUS, que tem sua origem na nova Constituição brasileira de 1989, bem como a regulação da saúde privada em 1998 são, em perspectiva histórica, bem recentes. O nosso SUS é o único no mundo que tem abrangência para mais de 200 milhões de habitantes e todos aqueles que estiverem em solo nacional, bem como atenção à saúde, com a criação de políticas para a geração de saúde, vigilância epidemiológica, atenção primária, secundária e especializada, com a cobertura gratuita de medicamentos de atenção básica e também 
de alto custo. O subfinanciamento do setor público de saúde, bem como as alocações orçamentárias realizadas sem a estrutura adequada, vem gerando diversas iniquidades aos usuários do sistema. Essas iniquidades vão desde a cobertura de medicamentos sem grandes diferenciais clínicos versus terapias-padrão, como a construção de hospitais para a ampliação da assistência sem prévio estudo da rede assistencial.

$\mathrm{O}$ amadurecimento que os autores comentam neste trabalho visa reforçar a trajetória que já vem se realizando na estrutura de entrada e acompanhamento do paciente na utilização dos serviços de saúde, mas também reforçar e criar no vazio existencial a estratégia de desospitalização como forma de otimização de custos e de continuidade/devolução do paciente do sistema hospitalar para a atenção domiciliar por meio dos serviços de atenção domiciliar e programa de Melhor em Casa.

\section{Referências bibliográficas}

Braga Neto FC, Barbosa PR, Santos IS, Oliveira CMF. Atenção hospitalar: evolução histórica e tendências. In: Giovanella L, Escorel S, Lobato LVC, Noronha JC, Carvalho Al, orgs. Políticas e Sistema de Saúde no Brasil. 2a ed. rev. e amp. Rio de Janeiro: Editora Fiocruz; 2012.

Brasil. Ministério da Saúde. 2019. Available from: https://www.saude.gov.br/ acoes-e-programas/melhor-em-casa-servico-de-atencao-domiciliar/ dados-e-informacoes.

Brasil. Ministério da Saúde. Portaria no 825, de 25 de abril de 2016. Redefine a Atenção Domiciliar no âmbito do Sistema Único de Saúde (SUS) e atualiza as equipes habilitadas. Brasília: Ministério da Saúde; 2016.

Brasil. Ministério da Saúde. Secretaria de Atenção à Saúde. Departamento de Atenção Básica. Caderno de atenção domiciliar v. 1. Brasília: Ministério da Saúde; 2012.

Brasil. Portaria n 3.390, de 30 de dezembro de 2013. Institui a Política Nacional de Atenção Hospitalar (PNHOSP) no âmbito do SUS, estabelecendo-se as diretrizes para a organização do componente hospitalar da Rede de Atenção à Saúde. Diário Oficial da União 30 dez. 2013.
Carvalho MSN, Menezes LA, Cruz Filho AD, Maciel CMP. Desospitalização de crianças com condições crônicas complexas: perspectiva e desafios. Rio de Janeiro: Eldorado; 2019.

CNES - Cadastro Nacional de Estabelecimentos de Saúde. Consulta Estabelecimento. Brasília: MS. Available from: http://cnes.datasus.gov.br/. Access on: Aug 10, 2019.

Mendes EV. Os grandes desafios do SUS. Salvador: Instituto de Saúde Coletiva/Universidade Federal da Bahia (ISC/UFBA), Casa da Qualidade Editora; 2001.

Oliveira Neto AV, Dias MB. Atenção Domiciliar no Sistema Único de Saúde (SUS): o que representou o Programa Melhor em Casa? Divulg Saúde Debate. 2014;51:58-71.

Panorama. Publicação da Anahp - Associação Nacional de Hospitais Privados - maio/junho 2015.

Pereira Júnior N [tese de doutorado]. Política e gestão em hospitais universitários federais. Campinas: Unicamp; 2018.

Ramalho G. Brasil perde 34 mil leitos hospitalares no SUS em oito anos. [Globonews]. 2018. [cited 1 Mar 2020]. Available from: https://g1.globo. com/ciencia-e-saude/noticia/brasil-perde-34-mil-leitos-hospitalares-dosus-em-oito-anos.ghtml. Access on: Feb 2, 2020.

SIH - Sistema de Informações Hospitalares. RD - AlH Reduzida. Brasília: MS. Available from: ftp://ftp.datasus.gov.br/dissemin/publicos/ SIHSUS/200801_/dados/. Access on: Aug 10, 2019.

Silva KL, Sena RR, Castro WS. A desospitalização em um hospital público geral de Minas Gerais: desafios e possibilidades. Rev Gaúcha Enferm. 2017;38(4):e67762.

Silva KL, Sena RR, Seixas CT, Feuerwerker LCM, Merhy EE. Atenção domiciliar como mudança do modelo tecnoassistencial. Rev Saúde Pública. 2010;44(1):166-76.

Tanaka OY, Ribeiro, EL. Avaliação de implantação de programas nacionais. In: Tanaka OY, Ribeiro EL, Almeida CAL. Avaliação em saúde: contribuições para incorporação no cotidiano. 1a ed. Rio de Janeiro: Atheneu; 2017.

Teixeira CF, organizadora. Planejamento em saúde: conceitos, métodos e experiências. Salvador: EDUFBA; 2010. 